\title{
Entre évaluation formulaïque et résumé généralisant : le rôle de la dislocation à droite dans la clôture d'épisodes conversationnels
}

\author{
Anne-Sylvie Horlacher \\ Université de Neuchâtel \\ anne-sylvie.horlacher@unine.ch
}

\section{Introduction}

Cette étude s'intéresse à la façon dont les locuteurs font intervenir la dislocation à droite (désormais DD) ${ }^{1}$ comme dispositif syntaxique dans les phases de clôture. Après un bref encadrement théorique sur le concept de closing pour clarifier à quel type de clôture nous nous référons, nous tenterons d'illustrer comment une structure marquée syntaxiquement contribue à la clôture d'épisodes conversationnels.

Si certains travaux antérieurs ont souligné l'implication de la DD dans l'organisation du discours (par ex. Ashby 1988, 1994), peu de chercheurs se sont véritablement attaché à décrire les fonctions récurrentes qu'elle accomplit dans l'interaction. Les études syntaxiques (par ex. les travaux du Groupe Aixois de Recherche en Syntaxe, Blasco-Dulbecco, 1999) ont surtout insisté sur la nature du lien et les contraintes grammaticales régissant l'élément détaché, tandis que les approches fonctionnalistes-discursives (Givón, 1984 ; Lambrecht, 1994, 2001) ont avant tout analysé la disloquée à droite par rapport à sa structure informationnelle. Des recherches portant sur le français ont récemment montré que cette structure est impliquée dans l'organisation des tours de parole (lorsqu'elle sert à constituer une deuxième place de transition après une absence de relève de la part de l'interlocuteur), de même que dans la gestion des positionnements mutuels (notamment en permettant de recueillir un accord «rehaussé » [upgraded agreement]), contribuant ainsi à l'accomplissement de certaines activités pratiques.

Dans la lignée des recherches qui tentent d'articuler les dimensions grammaticale et interactionnelle des constructions linguistiques, nous nous proposons de décrire le fonctionnement des DD en position séquentielle de clôture, en payant une attention particulière au déroulement des activités de discours. Notre cadre théorique est celui de la linguistique interactionnelle (Ochs, Schegloff \& Thompson, 1996 ; Selting \& Couper-Kuhlen, 2001 ; Ford, Fox \& Thompson, 2002 ; Hakulinen \& Selting, 2005), issue de l'analyse conversationnelle d'inspiration ethnométhodologique (Sacks, 1992). Une telle approche envisage les constructions linguistiques comme des ressources auxquelles les participants recourent dans le déploiement de l'interaction pour accomplir des activités sociales.

A partir d'entretiens semi-directifs en français mettant en scène un(e) enquêteur/trice, accompagné(e) soit d'enseignants soit d'élèves invités à se prononcer sur le bilinguisme, nous décrirons donc les ressources grammaticales, lexicales et prosodiques auxquelles les participants recourent de façon récurrente pour arriver à clôturer conjointement une séquence conversationnelle. Si la clôture se révèle bien être l'une des tâches accomplies par la DD, cela confirmera l'importance que revêt ce format syntaxique pour l'organisation séquentielle et interactive.

\section{La notion de clôture en analyse conversationnelle}

La notion de clôture connaît des utilisations différentes au sein de la communauté conversationnelle et interactionniste. Par conséquent, il est important de clarifier brièvement quel est le type de clôture auquel nous nous référons lorsque nous évoquons ce terme et quels sont les positionnements séquentiels que nous prenons en compte dans l'analyse.

a) En analyse conversationnelle, le terme de clôture a d'abord été employé pour décrire la dernière phase d'une rencontre sociale, pendant laquelle les interlocuteurs ajustent réciproquement leurs tours de parole 
de manière à pouvoir terminer l'interaction. Dans ce sens, ce terme est utilisé pour décrire la clôture d'une activité sociale, que ce soit un appel téléphonique (Schegloff \& Sacks, 1973 ; Button, 1987, 1990), une rencontre en face-à-face, une interaction de service (Aston, 1992), etc. En d'autres termes, l'unité qui est ainsi clôturée est "a single conversation" (Schegloff \& Sacks, 1973 : 288-293).

b) Mais Schegloff \& Sacks (1973:292) reconnaissent que le terme est également utilisé pour décrire une clôture topicale ("topic closure"). Ainsi, il existe une deuxième acception de la notion de clôture en analyse conversationnelle, qui est liée à la gestion des topics, c'est-à-dire aux "thèmes" que les interlocuteurs traitent dans la conversation. ${ }^{2}$ Il faut toutefois distinguer deux types de topics : les topics délimités - dotés d'une introduction marquée et d'une clôture reconnaissable (autrement dit, avec des frontières claires), et les topics graduels, qui se modifient par glissements progressifs ("topic shading", Schegloff \& Sacks 1973 ; "stepwise mouvement", Sacks 1992 ; "stepwise transition", Jefferson, 1984). Si le passage d'un topic à l'autre se fait par une clôture plus ou moins marquée du premier topic, il sera alors aisé de localiser des lieux qui en délimitent explicitement les contours initiaux et finaux. Dans cette optique, la notion de clôture est utilisée pour décrire la transition d'un topic à l'autre dans la conversation.

c) Enfin, le concept de clôture est également utilisé par les chercheurs pour décrire les phases de l'interaction pendant lesquelles les interlocuteurs terminent une activité conversationnelle. On parlera alors dans ce cas de la clôture d'une séquence conversationnelle (cf. Schegloff, 1990 ; Mondada, 2006). Il est important de souligner que le concept de séquence est différent de celui de topic. Il se peut que la clôture d'une séquence coïncide avec une clôture topicale, mais pas nécessairement. Ainsi, un topic peut être modifié durant le déploiement d'une même séquence sans que l'intégrité structurelle de la séquence soit compromise. Au terme de séquence, Koroljia \& Linell (1996: 800) préfèrent celui d'épisodes, qui sont perçus comme des événements discursifs s'articulant autour d'une trajectoire topicale cohérente ou d'une activité commune ("discursive events or action sequences, each delimited from a prior and subsequent discourse and internally bound together by a coherent topical trajectory and/or common activity"). C'est la raison pour laquelle nous employerons parfois aussi le terme d'épisode conversationnel pour décrire l'unité ainsi clôturée par les participants à l'interaction.

Pour l'étude présentée ici, nous nous intéresserons avant tout aux clôtures du type c) en décrivant les ressources que les locuteurs déploient de manière régulière pour arriver à clôturer conjointement une séquence conversationnelle.

\section{La DD comme ressource syntaxique dans les clôtures de séquence}

\subsection{Les DD intervenant sous la forme d'une évaluation formulaïque}

Afin de donner un premier aperçu du phénomène, nous allons aborder brièvement deux exemples contenant chacun une DD intervenant sous la forme d'un commentaire évaluatif à la fin d'une séquence conversationnelle. Les DD de ce type présentent un terme axiologique dans leur clause-matrice et fournissent ainsi une sorte d'évaluation générale qui vient résumer la séquence avant le passage à une autre activité.

L'extrait 1 a été recueilli lors d'un entretien réunissant un enquêteur (Q) et différents enseignants bilingues invités à se prononcer sur leurs expériences du bilinguisme dans leur milieu professionnel et dans la vie de tous les jours. Le passage ci-dessous restitue la fin (provisoire) de la conversation entre $\mathrm{Q}$ et le locuteur $\mathrm{B}$ - un professeur de géographie - qui vient d'expliquer comment il enseignait sa matière en italien dans une région francophone : 


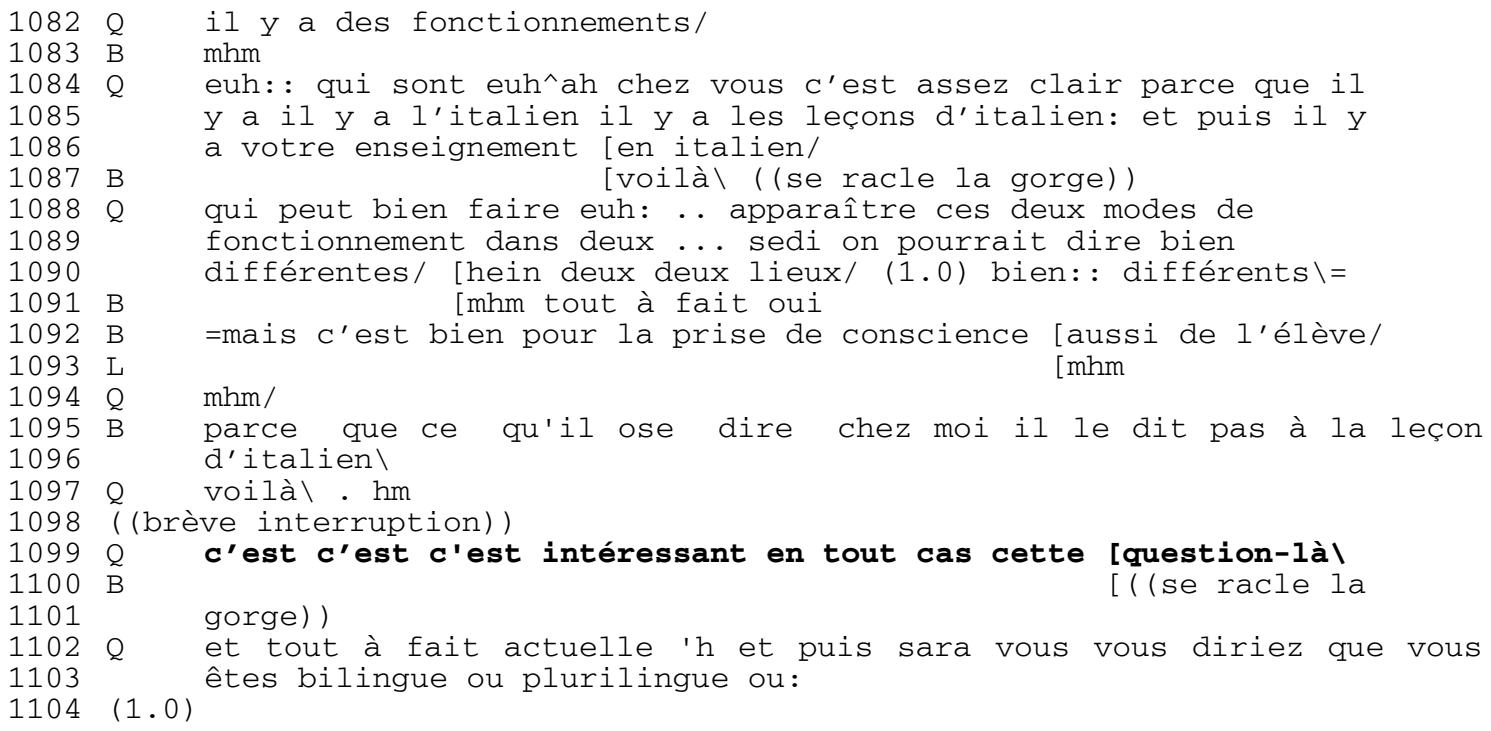

Aux lignes 1082 à 1090, l'enquêteur (Q) est en train de commenter les propos que B a formulés aux tours précédents. $\mathrm{B}$ affiche son accord avec les commentaires de l'enquêteur à travers deux acknowledgments aux lignes 1087 (voilà) et 1091 ( $m$ hm tout à fait oui). A la ligne 1092, il formule un tour qui est configuré comme un ajout (mais c'est bien pour la prise de conscience aussi de l'élève/) qui reçoit aux lignes 10931094 deux manifestations d'accord minimales. Après cet ajout, B intervient de nouveau avec un tour (11.1095-1096), présenté comme une explication (un account) de ce qu'il vient de dire au tour précédent (1.1092). A la ligne 1097, c'est l'enquêteur qui formule un tour d'accord (voilà . hm), qui est suivi d'une évaluation (ou assessment) sommaire c'est c'est intéressant en tout cas cette question-là $\backslash$ (1.1099) qui clôt définitivement le topic et la séquence dédiée au locuteur B. Le tour de Q en 1099 se caractérise par une structure syntaxique marquée, à savoir une DD, qui intervient visiblement dans une phase de clôture, puisque à la ligne 1102-3, l'enquêteur inaugure effectivement une nouvelle séquence en sélectionnant une autre personne (sara) comme prochaine interlocutrice.

Dans le deuxième extrait, la DD conclut un épisode conversationnel pendant lequel des élèves (âgés de 10 à 13 ans) ont été invités à raconter des anecdotes personnelles à l'enquêtrice sur leur utilisation d'une autre langue que le français dans la vie de tous les jours. Cette activité - qui dure depuis plus de 10 minutes - est conclue par l'enquêtrice en 1372 et 1373 avec un commentaire évaluatif global : c'est très intéressant tout ce que vous me racontez-là en tout cas :

\section{Exemple 2 (FNRS F)}

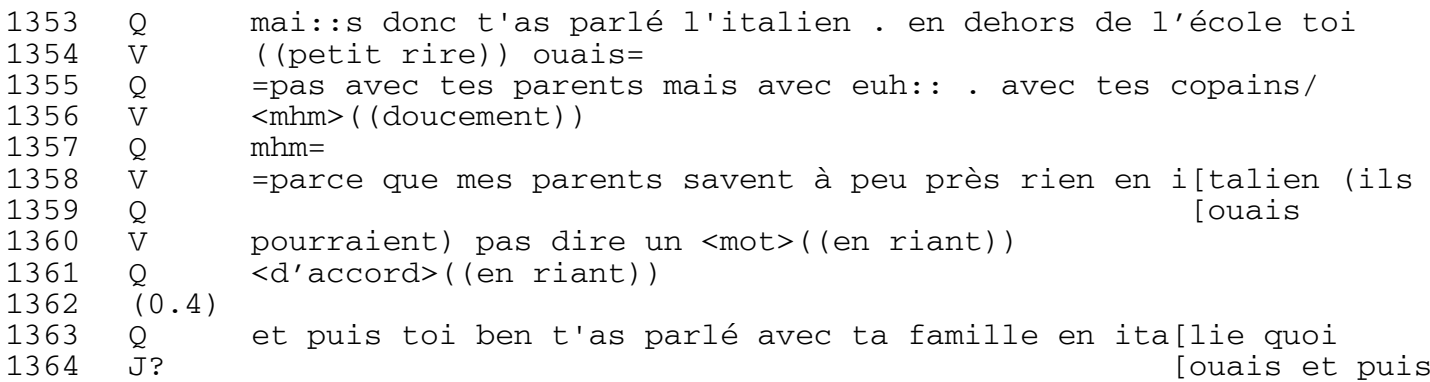




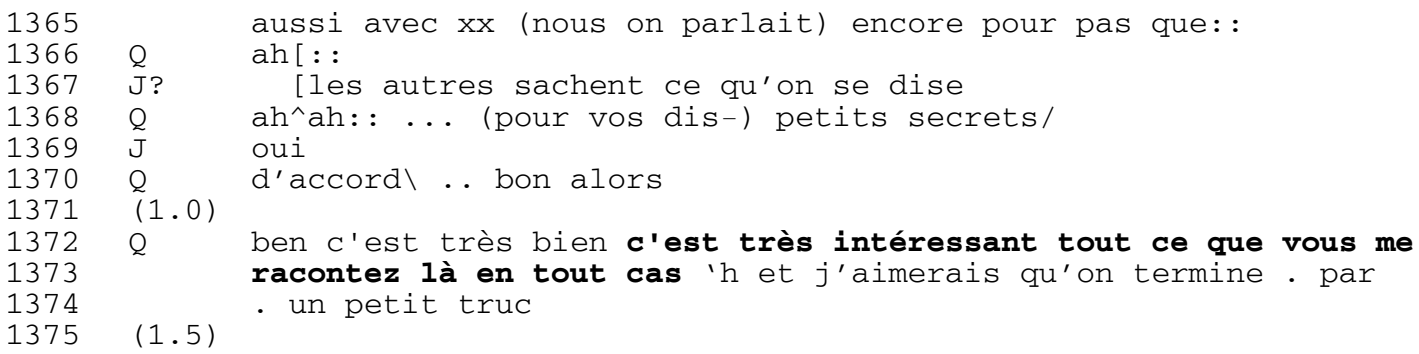

Dans cet exemple, le recours à la structure disloquée à droite constitue une ressource qui contribue à réaliser la clôture de l'activité dans laquelle les participants sont engagés. D'autres marques - présentes tout au long de l'extrait - indiquent que la séquence touche à sa fin et ne fera plus l'objet de développements ultérieurs. En effet, les élèves apportent massivement des marques de ratification aux propos de l'enquêtrice. En 1354 et 1356, l'élève V manifeste son accord de façon minimale par un ouais puis un $\mathrm{mhm}$, avant de livrer un dernier account (11.1358-1360) pour clarifier le sens de ses propos à l'enquêtrice. En 1361, cette dernière produit le marqueur de clôture d'accord, avant de s'adresser à une autre élève, dans une demande de ratification qui semble elle aussi résumer des propos échangés précédemment: et puis toi ben t'as parlé avec ta famille en italie quoi. Julie ratifie les propos de l'enquêtrice en les précisant (11.1364-5-1367). En 1370, l'enquêtrice formule un d'accord conclusif. Après une pause d'une seconde, elle produit une première évaluation de la discussion avec le terme axiologique bien: ben cest très bien. Puis, tout en fournissant une seconde évaluation positive de la séquence déroulée jusqu'ici - cette fois au moyen d'une structure disloquée à droite - l'enquêtrice s'oriente vers un autre type d'activité : j'aimerais qu'on termine par un petit truc (11.1373-4).

Malgré la brièveté des analyses, on note que la DD que l'enquêteur produit à la ligne 1099 dans l'extrait 1 , de même que celle de l'extrait 2 interviennent toutes les deux sous la forme d'une évaluation formulaïque pour clore une séquence conversationnelle. En produisant ces DD, les locuteurs n'apportent pas de nouveaux éléments relatifs au déroulement de la séquence, mais ils évaluent et résument l'information empirique fournie précédemment.

\subsection{Les DD survenant à la fin d'une argumentation ou d'un raisonnement : bilan généralisant et attente de ratification}

Issu du même corpus, l'exemple ci-dessous réunit quatre élèves qui - avant l'extrait reproduit - ont dû représenter par un dessin comment ils s'imaginaient le fonctionnement d'un cerveau de bilingue afin de le décrire à l'enquêtrice :

\section{Exemple 3 (FNRS F)}

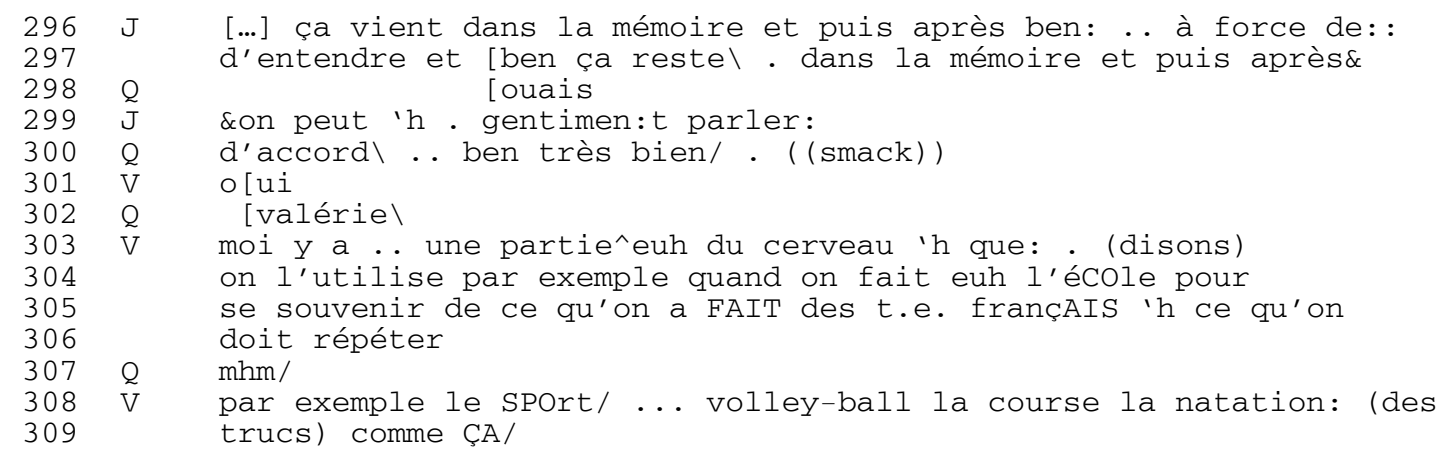




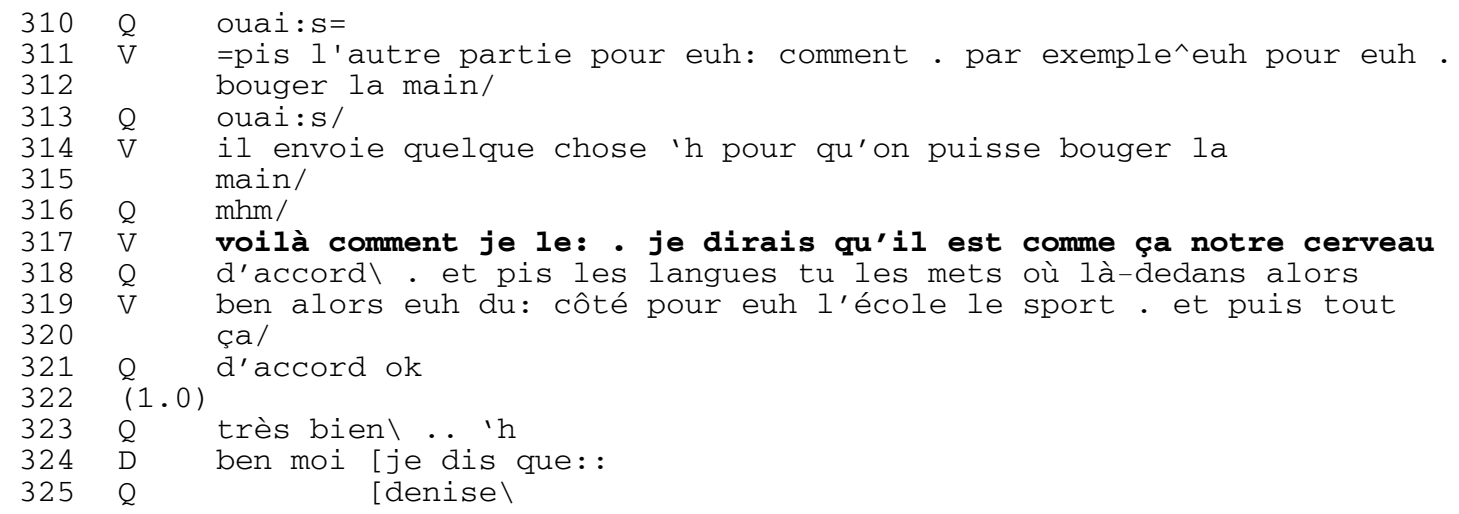

Les lignes 296 à 300 marquent la fin de l'intervention de l'élève J, qui a été conclue par Q en 300 par un d'accord conclusif et la formule ben très bien.

En 302, l'enquêtrice énonce le prénom de valérie/, sur un ton très montant, qui annonce l'intervention de l'élève suivante. Pendant toute l'explication de son dessin par Valérie, l'enquêtrice se contente d'énoncer des particules de feed-back comme mhm (1.307), ouai:s (1.310), ouai:s/ (1.313), mhm/ (1.316), ce qui traduit assez clairement l'abandon de l'espace discursif de l'enquêtrice à l'élève.

A la ligne 317 , l'élève $\mathrm{V}$ initie son tour par un voilà $\$-massivement employé dans les séquences de clôture - avant d'utiliser une formule conclusive sous forme de disloquée à droite je dirais qu'il est comme ça notre cerveau, qui marque la fin de son explication. En quelque sorte, la DD clôt ici son raisonnement et son activité participante. Mais si la structure disloquée à droite contribue à clore l'activité de raisonnement de Valérie et à rendre reconnaissable qu'elle n'a rien d'autre à ajouter, son effet clôturant est également perçu par l'enquêtrice, puisque celle-ci s'auto-sélectionne en 318, exhibant par là qu'elle a identifié une position séquentielle adéquate pour la prise de parole. Elle énonce en effet un d'accord $\Lambda$ avec une intonation descendante traduisant, elle aussi, une orientation vers la clôture. En d'autres termes, cette manifestation d'accord suggère que la proposition de clôture que Valérie projette en 317 est ratifiée par l'enquêtrice.

Cependant, après la manifestation d'accord et une légère pause, le tour de l'enquêtrice fait apparaître le marqueur d'articulation et pis qui accompagne l'ouverture d'un autre segment de discours : et pis les langues tu les mets où là-dedans alors (1.318). Par la formulation de cette première partie de paire adjacente, l'enquêtrice suspend la clôture initiée précédemment. Il est intéressant que cette réouverture s'effectue ici par le biais d'une dislocation à gauche (DG), qui sert typiquement à réintroduire un référent (ici, les langues) qui n'est pas dans l'avant-plan de la conscience des interlocuteurs ou à retourner à un topic antérieur (Duranti \& Ochs, 1979; Lambrecht, 2001; Pekarek Doehler, 2001). En effet, l'élève Valérie a oublié de placer les langues dans son dessin, qui constituait l'un des enjeux de l'exercice. La clôture est donc retardée par une demande de précision de la part de l'enquêtrice, qui trouve réponse en 319-320 - réponse que l'enquêtrice ratifie dans le tour suivant par un d'accord ok. Après une pause d'une seconde, l'enquêtrice apporte une nouvelle ratification à sa clôture précédente (très bien $\backslash 1.323$ ) qui marque la clôture effective de l'épisode conversationnel consacré à Valérie, et l'orientation vers une autre séquence. La prise de tour de l'élève Denise en 324, puis l'annonce - en chevauchement - par l'enquêtrice du prénom de Denise (1.325) initient l'ouverture de la phase suivante.

Dans l'extrait analysé, nous pouvons donc affirmer que la DD intervient dans un tour qui projette la clôture d'une séquence. Elle n'accomplit pas à elle seule la clôture, qui finira d'être achevée dans les tours qui suivent. Dans ce sens, la DD déploie ici prospectivement un effet conclusif, et les tours suivants participent à la clôture effective de la séquence. En d'autres termes, la clôture séquentielle est rarement réalisée uniquement à travers une DD. Nous avons mentionné divers facteurs co-occurrents qui contribuent également à sa réalisation. 
L'exemple suivant illustre lui aussi le cas d'une DD qui survient à la fin d'un raisonnement. Le passage met en scène un enquêteur et quatre adultes - tous enseignants de langue et bilingues - impliqués dans la même activité qu'illustrée dans l'exemple ci-dessus. Après avoir imaginé le cerveau d'un bilingue, chacun exprime son point de vue en commentant son dessin. Dans le passage sélectionné, le locuteur B et la locutrice L défendent deux lignes argumentatives différentes. B s'imagine le cerveau d'un bilingue comme un seul bocal (1.2367), alors que L insiste sur le fait que la réalité d'une personne bilingue est constamment tiraillée entre deux langues, ce qui résulte en deux bocaux distincts dans sa représentation du phénomène :

\section{Exemple 4 (FNRS B)}

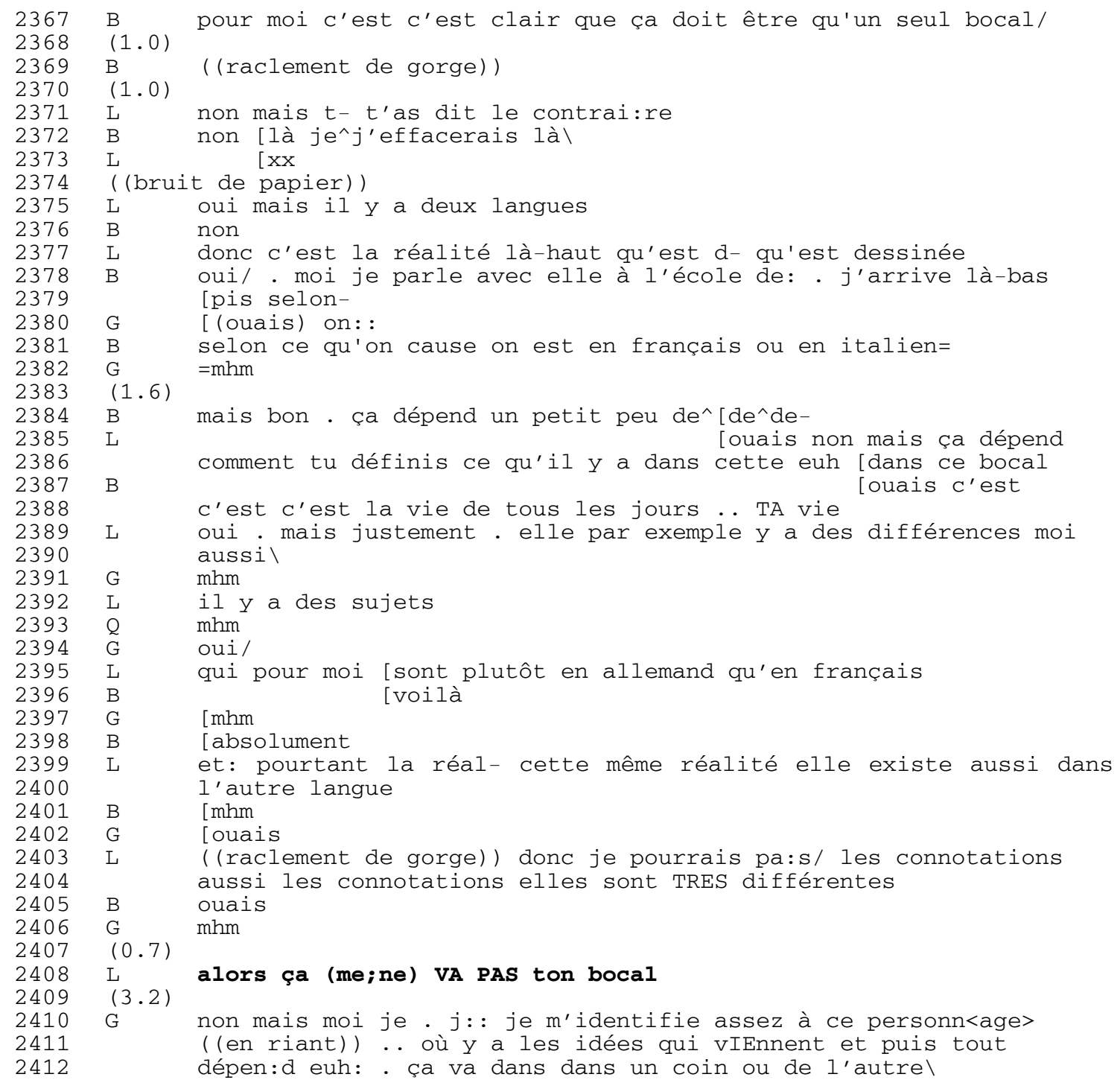

Dans cet extrait, le locuteur B et la locutrice L défendent leurs positions et leurs arguments en se situant soit en accord ou en désaccord avec le point de vue exprimé par l'un ou l'autre dans le tour précédent, ce qui explique les nombreux tours de parole commençant par des mais $(1.2384)$, non mais $(1.2371,1.2385)$ 
ou oui mais (1. 2375, 1. 2389), selon que la locutrice L réfute l'argument proposé par le locuteur B dans le tour précédent et vice-versa.

La DD survient à la ligne 2408 à la fin de l'argumentation de L : alors ça (me;ne) va pas ton bocal. Après une pause de plus de trois secondes qu'aucun des participants ne traite comme une opportunité pour prendre la parole, la locutrice G s'approprie le tour et dominera le floor conversationnel pour décrire à son tour son dessin en reprenant quelques-uns des arguments développés à la fois par L et par B.

Ainsi, les exemples 3 et 4 fournissent une illustration supplémentaire de DD en position séquentielle de clôture. Ces DD surviennent à la fin d'une argumentation ou d'un raisonnement sous la forme d'un commentaire généralisant qui réduit considérablement la complexité de l'argument traité. Sans apporter de nouvel argument à l'explication fournie, la DD résume les propos échangés dans le discours précédent.

En cela, ces DD « conclusives » sont à rapprocher des expressions figurées de Drew \& Holt (1995, 1998, 2005: 35) «fomulaic and fixed in composition ». Sans toutefois évoquer la structure disloquée à droite, Drew \& Holt (1998 : 495) notent que ces formules sont récurrentes dans les séquences de transition topicale (« in topic transition sequences, and specifically in the turn where a topic is summarized, thereby initiating the closing of a topic ») et que souvent elles résument le topic traité dans les tours précédents («serve as summary assessments of what has been reported»). De plus, Drew \& Holt (1998 : 502-3) observent qu'elles permettent aux interlocuteurs «[to] move away from, or step out of, their report of empirical details ». En bref, la fonction principale de ce type d'expressions réside dans leur capacité à réduire la complexité en faisant abstraction des détails. C'est exactement cet effet généralisant - qui permet de s'éloigner des faits empiriques pour formuler une sorte de maxime à laquelle on attribue une validité générale - que nous évoquons pour expliquer l'effet clôturant que les locuteurs semblent attribuer à la DD. Dans ce sens, les DD examinées évoquent aussi les formules routinières, proverbiales ou aphoristiques de Schegloff \& Sacks (1973 : 306): "proverbial or aphoristic formulation[s] of conventional wisdom which can be heard as the 'moral' or the 'lesson' of the topic being thereby possibly closed ».

Dans le dernier exemple que nous analyserons, l'enquêtrice interroge les élèves sur la place que doit prendre l'étude des langues à l'école par rapport aux autres branches. Pour enrichir le débat, elle leur demande de commenter des citations qu'ils ont préalablement choisies sur des petites cartes. Le passage ci-dessous concerne essentiellement la réaction de Valérie :

\section{Exemple 5 (FNRS F)}

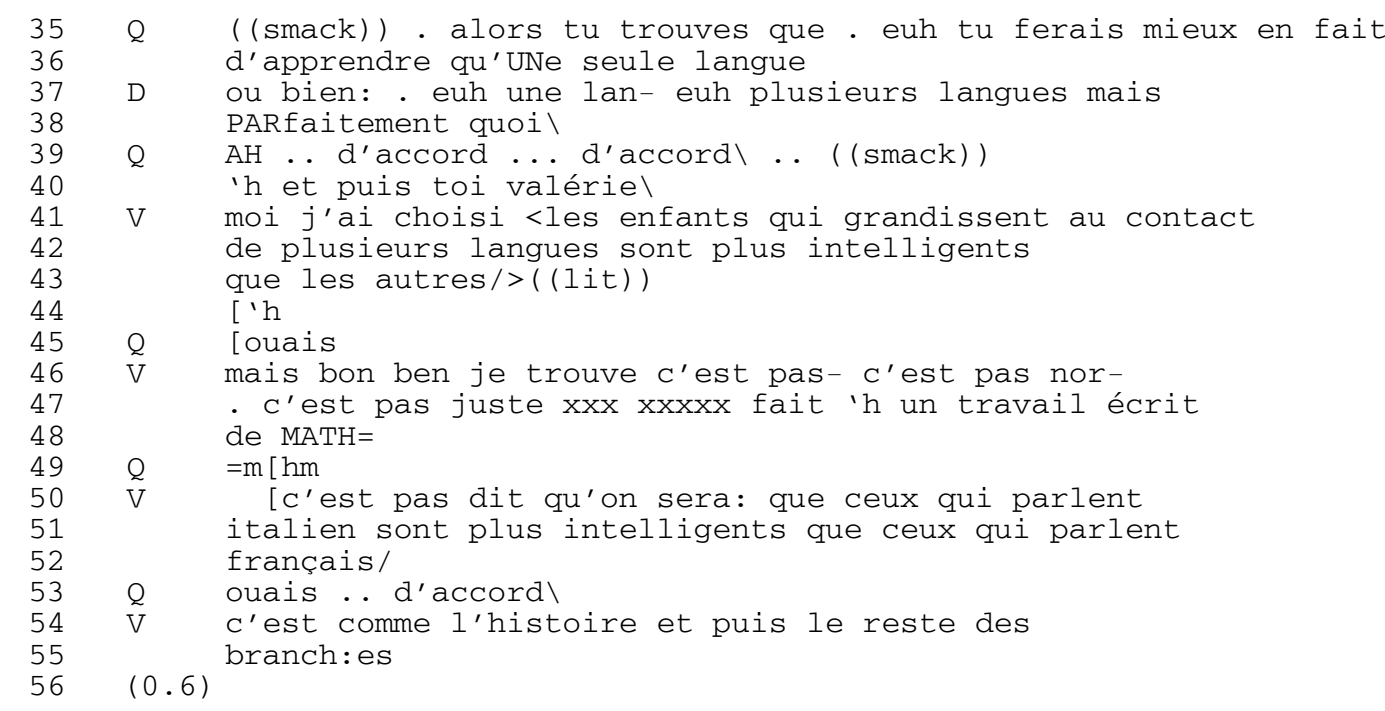




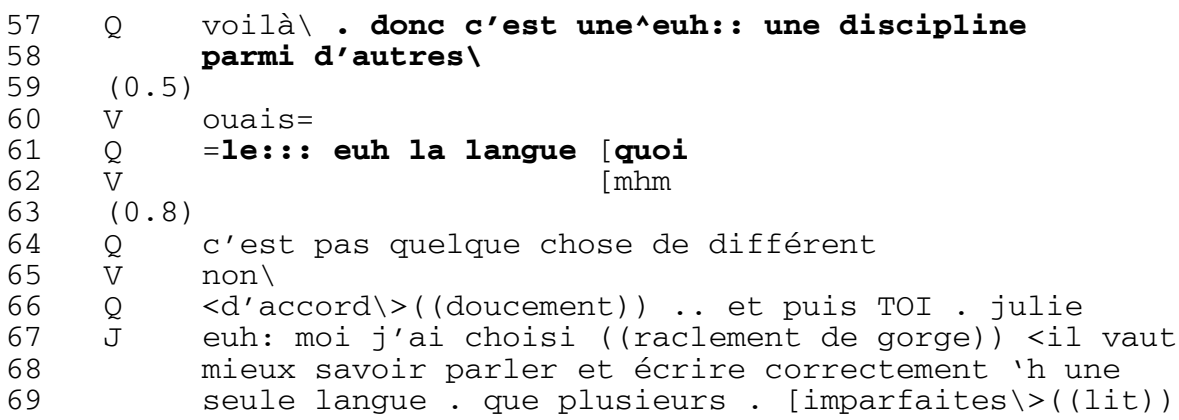

Les lignes 35 à 39 reproduisent la fin de l'intervention de l'élève David, qui a commenté l'affirmation : "il vaut mieux savoir parler et écrire correctement une seule langue que plusieurs imparfaitement". L'enquêtrice est engagée dans une activité qui consiste à reformuler les propos de l'élève, afin d'en tirer une conclusion qu'elle lui soumet à approbation : ((smack)). alors tu trouves que. euh tu ferais mieux en fait d'apprendre qu'UNe seule langue (11.35-36). Sa question comporte une forte accentuation sur le nombre dans $U N e$ seule langue, ce qui traduit peut-être aussi une demande de clarification. David rectifie dans le tour suivant : une lan-euh plusieurs langues mais PARfaitement quoi (11.37-8). L'enquêtrice réagit dans le tour suivant avec une forte interjection $A H$ (1.39), manifestant par là qu'il y avait effectivement un problème de compréhension mais qu'il est à présent résolu, ce que traduit la présence répétée des d'accord (1.39). L'interaction peut alors se poursuivre avec une autre élève : ' $h$ et puis toi valérie (1.40).

A partir de la ligne 41, c'est donc au tour de Valérie d'expliquer et de commenter la phrase qu'elle a choisie. Elle essaie notamment de démentir (c'est pas juste, 1.47) l'affirmation selon laquelle les enfants qui grandissent au contact de plusieurs langues sont plus intelligents que les autres (1l.41-43). Son argument est que la connaissance d'une autre langue que le français n'aidera pas forcément un élève à mieux réussir un travail écrit de maths : c'est pas dit [...] que ceux qui parlent italien seront plus intelligents que ceux qui parlent français/ (11.50-52). L'enquêtrice manifeste un accord à la contribution de Valérie en 53. Cette dernière conclut son intervention en affirmant que c'est comme l'histoire et puis le reste des branch:es (11.54-55). A la ligne 57, l'enquêtrice marque la fin de l'argumentation de Valérie par un voilà $\backslash$ conclusif. Puis, comme pour David précédemment, elle formule un énoncé-bilan qui offre une synthèse des propos de Valérie, mais qui en même temps ré-ouvre la séquence conversationnelle consacrée à cette élève, alors qu'elle semblait avoir été clôturée dans les tours précédents : donc c'est une $e u h::$ une discipline parmi d'autres $\backslash$ (11.57-58). Une pause survient en 59 avant le ouais (1.60) que l'élève livre en guise de ratification à l'intervention de l'enquêtrice. Devant ce qu'elle interprète comme une absence d'alignement - puisque produit quasi simultanément - l'enquêtrice enchaîne avec une poursuite de la structure initiée en 57-58. Cet ajout est précisément le référent plein à associer à l'article démonstratif $c^{\prime}$ de son tour précédent. En d'autres termes, le format syntaxique qui résulte de son extension de tour est une DD. Puis Valérie livre un deuxième acquiescement minimal $(\mathrm{mhm}, 1.62)$ peu avant la fin de cet ajout, en chevauchement avec l'enquêtrice. Le tour 63 accueille une nouvelle pause d'un peu moins d'une seconde, alors l'enquêtrice s'auto-sélectionne à nouveau pour reformuler à Valérie une demande qui paraphrase la précédente : c'est pas quelque chose de différent (1.64). Valérie enchaîne encore de façon minimale avec un non $(1.65)$. Comme les interventions de Valérie n'alimentent plus le débat, l'enquêtrice met un terme à la séquence consacrée à la réaction de Valérie avec un d'accord prononcé doucement sur un ton très descendant (1.66), puis poursuit avec un énoncé orienté vers l'élève suivante : et puis TOI . julie (1.66).

A nouveau, nous argumentons que la DD joue dans cet exemple un rôle résomptif. Nous constatons que le tour contenant la DD est en effet traité comme projetant la fin de la séquence conversationnelle consacrée à Valérie. Aux lignes 57-58 et 61, l'enquêtrice fournit au moyen de la DD une sorte de reformulation généralisante des propos de l'élève. Il est difficile de spéculer si cet énoncé récapitulatif projette un accord minimal ou s'il sollicite véritablement une élaboration de la part de Valérie. Il reste que la briéveté des tours de l'élève - qui n'apporte plus que des accords minimaux aux tours de Q - ainsi que 
la diminution du volume de la voix de l'enquêtrice marquent l'orientation des participants vers le fait que la réaction de l'élève touche à sa fin. La phase suivante peut ainsi être initiée : elle consistera dans l'annonce de l'élève suivante (julie) par l'enquêtrice avec une augmentation du volume de la voix.

\section{Conclusion}

La DD est un moyen syntaxique que les participants mettent en œuvre lorsqu'ils sont orientés vers la clôture d'une séquence conversationnelle. Si le recours à une structure disloquée ne représente qu'une manière parmi d'autres de clore une séquence, elle n'en constitue pas moins une ressource que les locuteurs font intervenir de manière régulière. En payant une attention particulière au placement séquentiel de cette construction dans le déroulement des activités de discours, les analyses ont mis en évidence que les DD interviennent souvent pour résumer une séquence sous la forme d'une évaluation formulaïque, recourant à des expressions figées relevant du sens commun. En outre, elles sont souvent utilisées par les locuteurs pour marquer la fin d'une argumentation ou d'un raisonnement et ainsi clore leur activité participante. Enfin, ces DD apparaissent fréquemment sous la forme d'un énoncé récapitulatif ou d'un commentaire global qui réduit la complexité des propos échangés (permettant de passer d'une description des faits empiriques à une généralisation), tout en cherchant à susciter l'accord des participants avant le passage à une autre séquence. Ainsi, ce dispositif - sans apporter de nouveaux éléments relatifs au topic ou à la séquence concernée - contribue cependant à la clôture, en évaluant et en résumant le discours précédent.

Ces observations - encore tout à fait exploratoires - nécessiteraient d'être élargies par l'étude d'autres structures syntaxiquement marquées (comme p.ex. les pseudo-clivées ou les topicalisées). D'autre part, il se profile le besoin de corroborer ces premières analyses par des études fondées sur des collections d'exemples plus amples.

\section{Références bibliographiques}

Ashby, W. J. (1988). The Syntax, Pragmatics, and Sociolinguistics of Left and Right Dislocations in French. Lingua, 75 (2-3), 203-229.

Ashby, W. J. (1994). An Acoustic Profile of Right Dislocations in French. Journal of French Language Studies, 4 (2), 127-45.

Aston, G. (1992). What it takes to close a service encounter. Bologna : Clueb.

Blasco-Dulbecco, M. (1999). Les dislocations en français contemporain. Etude syntaxique. Paris : Champion.

Button, G. (1987). Moving out of Closings. In Button, G. \& Lee, J. R. (éds.), Talk and Social Organisation. Clevedon : Multilingual Matters, 101-151.

Button, G. (1990). On varieties of closings. In Psathas, G. (éd.), Interaction Competence. Washington : University Press of America, 93-147.

Couper-Kuhlen, E. \& Selting, M. (2001). Studies in Interactional Linguistics. Amsterdam : Benjamins.

Drew, P. \& Holt, E. (1995). Idiomatic expressions and their role in the organization of topic transition in conversation. In Everaert, M. et al. (éds.), Idioms. Structural and psychological perspectives. Hillsdale NJ : Erlbaum, 117-132.

Drew, P. \& Holt, E. (1998). Figures of speech: Figurative expressions and the management of topic transition in conversation. Language in Society, 27, 495-522.

Duranti, A. \& Ochs, E. (1979). Left dislocation in Italian conversation. In Givón, T. (éd.), Discourse and syntax. New York : Academic Press, 377-416

Ford, C. E., Fox, B. A. \& Thompson, S. A. (2002). Constituency and the grammar of turn increments. In Ford, C. E., Fox, B. A. \& Thompson, S. A. (éds.), The Language of Turn and Sequence. Oxford : Oxford University Press, 14-38. 
Givón, T. (1984). Topic Continuity in Discourse: An Introduction. In Givón, T. (éd.), Topic Continuity in Discourse: A Quantitative Cross-Language Study. Amsterdam : Benjamins, 1-41.

Hakulinen, A. \& Selting, M. (2005). Syntax and Lexis in Conversation. Studies on the Use of Linguistic Resources in Talk-in-Interaction. Amsterdam : John Benjamins.

Holt, E \& Drew, P. (2005). Figurative Pivots: The Use of Figurative Expressions in Pivotal Topic Transitions. Research on Language and Social Interaction, 38 (1), 35-61.

Jefferson, G. (1984). On stepwise transition from talk about a trouble to inappropriately next-positioned matters. Structures of social action. In Atkinson, J. M. \& Heritage, J. (éds), Studies in conversation analysis. Cambridge : Cambridge University Press, 191-222.

Koroljia, N. \& Linell, P. (1996). Episodes: coding and analyzing coherence in multiparty conversation. Linguistics, $34,799-831$.

Lambrecht, K. (1994). Information structure and sentence form. Topic, focus, and the mental representations of discourse referents. Cambridge : Cambridge University Press.

Lambrecht, K. (2001). Dislocation. In Haspelmath, M., (éd.), La typologie des langues et les universaux linguistiques. Manuel international. Berlin : Walter de Gruyter, 1050-1078.

Mondada (1994). Verbalisation de l'espace et fabrication des savoirs. Approche lingusitique de la construction des objets de discours. Lausanne : Université de Lausanne.

Mondada, L. (2006). Participants' online analysis and multimodal practices: projecting the end of the turn and the closing of the sequence. Discourse Studies, 8, 117-129.

Ochs, E., Schegloff E. A. \& Thompson S. E. (éds) (1996). Interaction and Grammar. Cambridge : Cambridge University Press.

Pekarek Doehler, S. (2001). Dislocation à gauche et organisation interactionnelle. Marges Linguistiques, 177-194 (http://www.marges-linguistiques.com).

Sacks, H. (1992). Lectures on conversation. Oxford : Basil Blackwell.

Schegloff, E. A. \& Sacks, H. (1973). Opening up closings. Semiotica, 8 (3), 289-327.

Schegloff, E. A. (1990). On the organization of sequences as a source of "coherence" in talk-in-interaction. In Dorval, B. (éd.), Conversational organization and its development. Norwood NJ : Ablex Publishing Corporation, 52-77.

\section{Conventions de transcription}

1 intonation montante

1 intonation descendante

. .. ... pause courte, moyenne ou longue

(1.5) temps de pause indiqué en secondes et dixièmes de seconde

[ début du chevauchement

xxx segment inaudible

((rire)) commentaires du transcripteur

$<>\quad$ début et fin du segment auquel s'appliquent les commentaires du transcripteur

( ) transcription incertaine

\& continuation du même tour

$=\quad$ enchaînement rapide

video accent

exTRA augmentation de volume

: allongement syllabique

par- troncation

$\wedge \quad$ liaison 
ISBN 978-2-7598-0358-3, Paris, 2008, Institut de Linguistique Française

Discours, pragmatique et interaction

DOI $10.1051 / \mathrm{cmlf0} 193$

\author{
'h aspiration \\ h' expiration \\ $(($ smack $)) \quad$ ouverture de bouche
}

\begin{abstract}
${ }^{1}$ Nous entendons par DD une construction syntaxique dont un des éléments est détaché à droite, tout en étant représenté dans la clause principale par un pronom clitique, de sorte qu'il s'établit entre le constituant détaché et ce pronom un rapport dit de co-référence (cf. Lambrecht 2001). Selon la plupart des descriptions de la DD, l'élément détaché est typiquement un syntagme nominal, lexical ou pronominal. Cependant, nous n'excluons pas que d'autres éléments puissent figurer dans une DD (un verbe, un adjectif, etc.).

2 Mondada (1994 : 63) définit le topic comme "ce à propos de quoi le 'je' énonce son discours". Autrement dit, nous adoptons ici une définition du topic telle qu'elle est utilisée dans les approches conversationnalistes et interactives pour désigner ce sur quoi porte la conversation à un moment précis de l'interaction.
\end{abstract}

